\title{
LAS ENFERMEDADES NEOPLÁSICAS COMO CAUSA DE INCAPACIDAD PERMANENTE
}

\author{
A. ARES CAMERINO, B. SAINZ VERA (*), \\ J.C. MARCHENA APARICIO, M.L. SOTO PINO \\ Servicio de Prevención y Salud Laboral \\ Diputación Provincial de Cádiz \\ (*) Servicio de Medicina Interna \\ Hospital Universitario Puerto Real (Cádiz)
}

\section{RESUMEN}

El cáncer es la primera causa de mortalidad en hombres y la segunda en mujeres, tras las enfermedades cardiovasculares. Desde el punto de vista económico, los costes atribuibles a las enfermedades neoplásicas son principalmente indirectos (pérdida de productividad asociada a la mortalidad o a la incapacidad permanente).

Analizando las incapacidades permanentes producidas en la Diputación Provincial de Cádiz en el período 1990-2005, que fueron un total de 208 , se concluye, por orden de frecuencia, que las enfermedades neoplásicas se sitúan en tercer lugar $(17,87 \%)$, precedidas de las enfermedades osteomusculares $(24,15 \%)$ y de las enfermedades cardiovasculares $(23,67 \%)$. En cuanto a la pérdida de años e vida laboral, las enfermedades neoplásicas ocupan el segundo lugar con 11,24 años, detrás de las enfermedades psiquiátricas con 14,18 años.

Por sexos las causas de incapacidad permanente se distribuyeron por el siguiente orden decreciente. En mujeres: enfermedades osteomusculares $(39,08 \%)$, enfermedades neoplásicas $(19,54 \%)$ y enfermedades cardiovasculares $(16,09 \%)$. En hombres: enfermedades cardiovasculares $(28,93 \%)$, enfermedades neoplásicas $(16,53 \%)$ y enfermedades osteomusculares $(13,22 \%)$.

\section{PALABRAS CLAVES}

Cáncer, incapacidad.

\begin{abstract}
The cancer is the first cause of mortality in men and second in women, after the cardiovascular diseases. From the economic point of view, the costs attributable to the cancer diseases are mainly indirect (loss of productivity associated to mortality or the permanent incapacity). Analysing the produced permanent incapacities in the Provincial Delegation of Cadiz in period 1990-2005, that were a total of 208 , one concludes, by frequency order, which the cancer diseases are located thirdly $(17.87 \%)$, preceded of the diseases osteomusculares $(24.15 \%)$ and of the cardiovascular diseases $(23.67 \%)$. As far as the loss of years and labour life, the cancer diseases occupy the second place with 11.24 years, behind the psychiatric diseases with 14.18 years. By sexes the causes of permanent incapacity were distributed by the following decreasing order. In women: diseases osteomusculares $(39.08 \%)$, cancer diseases (19.54\%) and cardiovascular diseases (16.09\%). In men: cardiovascular diseases $(28.93 \%)$, cancer diseases $(16.53 \%)$ and diseases osteomusculares $(13.22 \%)$.
\end{abstract}

\section{KEY WORDS}

Cancer, incapacity. 


\section{INTRODUCCIÓN}

En el año 2003 fallecieron en España 96.257 personas por cáncer con un claro predominio de hombres (razón de sexo 1,7:1). Uno de cada tres hombres muere de enfermedades neoplásicas, mientras que en el sexo femenino es una de cada cinco mujeres. El cáncer es la primera causa de mortalidad entre hombres, y la segunda en las mujeres, tras las enfermedades cardiovasculares (1).

Globalmente, la incidencia de cáncer en los registros españoles de base poblacional se sitúa a nivel medio con respecto a Europa y al resto del mundo, siendo algo más baja en el sexo femenino. En todos los registros españoles, los tumores mas frecuentes son los de mama, colorectal y ginecológico entre las mujeres, y el de pulmón, colorectal, próstata y vejiga entre los hombres (2).

La incidencia del cáncer de pulmón es alta en el sexo masculino en casi todos los registros, y baja en el femenino, aunque es previsible que la tendencia se invierta. El cáncer de próstata tiene una incidencia baja en los registros especiales con respecto al resto de los países desarrollados, mientras que se puede considerar alta para el cáncer de vejiga.

La incidencia de cáncer colorectal, estómago e hígado en ambos sexos es de las más baja de nuestro entorno.

La incidencia de cáncer de mama en los registros especiales es baja si la comparamos con la de países de semejante nivel de desarrollo.

Se observa una tendencia descendente en la mortalidad por cáncer de mama, colon, testículo, útero y linfomas de Hodgkin, mientras que la tendencia es ascendente en los tumores de pulmón femenino, linfomas no Hodgkin, mieloma, melanoma maligno y páncreas. La mortalidad por cáncer colorectal y pulmón masculinos tiende a estabilizarse y la única tasa que aumenta de manera muy significativa es la de cáncer de pulmón femenino, sobre todo en el último decenio (3).

El estudio EURO CARE-3 (European Cancer Registry Study of Survival on Patients) que reúne un amplio grupo de investigadores europeos coordinados por el Instituto Nacional para el Estudio de las Causas de los Tumores, con sede en Milán (Italia) sitúan la supervivencia de los pacientes con cáncer en España en una situación comparable a la de los países mas desarrollados, situándose en determinadas localizaciones por encima de la media europea.
La supervivencia ha mejorado aproximadamente un $10 \%$ en la década de los noventa, y se espera que esta tendencia continúe para la mayoría de los tumores en las décadas venideras.

Con carácter general las enfermedades cardiovasculares en primer lugar y las enfermedades neoplásicas en segundo lugar, son las que producen mayor mortalidad en los países industrializados. Por ello, cada vez son mas los estudios que tienen como objetivo el realizar un análisis socioeconómico de los costes de dichas patologías $(4,5)$.

Los costes directos son los que se refieren directamente al consumo de recursos del sistema de atención sanitaria (atención hospitalaria, hospitalizaciones, atención primaria, fármacos, etc); y los costes indirectos los relacionados con la disminución o pérdida de productividad debida a la mortalidad prematura y a la incapacidad laboral atribuible a una patología determinada

Según diversos estudios $(4,6-7)$ en el caso de las enfermedades neoplásicas el mayor porcentaje de los costes son de tipo indirecto. La pérdida de productividad asociada a la mortalidad o incapacidad permanente suele estimarse como la producción que se habría llevado a cabo desde el momento de la declaración de incapacidad permanente o del fallecimiento, hasta el momento de la edad reglamentaria de jubilación, 65 años. La pérdida de productividad asociada a la morbilidad tiene dos manifestaciones básicas: una es la derivada del absentismo por enfermedad y otra es la reducción de productividad de un trabajador/a enfermo o incapacitado temporalmente para su trabajo.

Con estos antecedentes se propone realizar un estudio que tiene como objetivo el conocer importancia de las enfermedades neoplásicas como causa de incapacidad permanente (total o absoluta) comparándola con las otras causas, entre la población trabajadora de la Diputación Provincial de Cádiz en el período comprendido entre 1990 y 2005.

\section{MATERIAL Y MÉTODO}

\section{Diseño del estudio:}

Estudio descriptivo transversal. Se analizaron todas las incapacidades permanentes, tanto en su grado de total como de absoluta, que durante el período comprendido entre el 1 de enero de 1990 y el 31 de diciembre de 2005 se produjeron entre la población trabajadora de la Diputación Provincial de Cádiz. 


\section{Ambito:}

La Diputación Provincial de Cádiz, Corporación de Administración Local, tiene una población trabajadora de alrededor de 1600 trabajadores, con un claro predominio femenino (alrededor del 60\%). Sus centros de trabajos, alrededor del centenar, se distribuyen por todo el ámbito provincial. Las actividades laborales que en ellos se realizan son muy diversas, y van desde trabajos meramente administrativos, hasta actividades asistenciales infantiles, de mayores, drogodependencias, actividades agrícolaganaderas, educativas, medioambientales, mantenimiento de carreteras, turísticas, etc

\section{Metodología:}

En todos los casos se analizaron las siguientes variables: tipo de incapacidad (total o absoluta), edad, sexo, antigüedad en la empresa y diagnóstico o causa principal de la incapacidad.

Para el procesamiento de os datos se creó una base de datos con Microsoft Access. Los sencillos cálculos estadísticos se realizaron utilizando Microsoft Excell.

\section{RESULTADOS}

Durante el período estudiado, en la Diputación Provincial de Cádiz, se resolvieron un total de 208 expedientes de Incapacidad Permanente: 121 hombres $(58,17 \%)$ y 87 mujeres $(41,83 \%)$. La media de edad de la población laboral incapacitada fue de 55,45 años (DS 4,32), no existiendo diferencia significativa por sexos.

Los datos del análisis de las variables edad, sexo, antigüedad en la empresa y pérdida de vida laboral (diferencia entre la edad obligatoria de jubilación y la edad en la que se resolvió el expediente de incapacidad) según los principales grupos diagnósticos se exponen en la TABLA 1

Del presente estudio se concluye que la primera causa de incapacidad permanente, en la población laboral estudiada fueron las enfermedades osteomusculares, seguidas de las enfermedades cardiovasculares y de las enfermedades neoplásicas.

De los resultados obtenidos en el presente estudio podemos extraer las siguientes conclusiones:

Tabla 1. Expedientes de Incapacidad Permanente. Diputación Provincial de Cádiz (1990-2005). Análisis por edad, sexo, antigüedad, pérdida de vida laboral y grupo diagnóstico.

\begin{tabular}{|c|c|c|c|c|c|c|c|c|}
\hline $\begin{array}{c}\text { Grupo } \\
\text { diagnóstico }\end{array}$ & Total & H & M & $\begin{array}{c}\text { Media } \\
\text { Edad }\end{array}$ & H & M & Antigüedad & $\begin{array}{c}\text { Pérdida vida } \\
\text { laboral }\end{array}$ \\
\hline
\end{tabular}

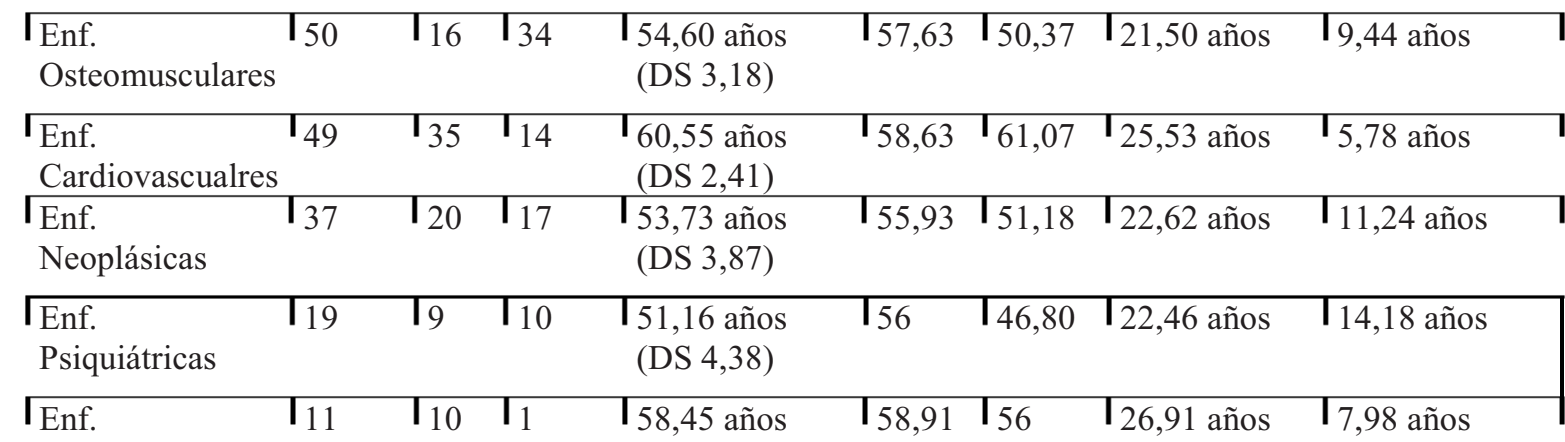

Respiratorias (DS 3,43)

\begin{tabular}{|c|c|c|c|c|c|c|}
\hline $\begin{array}{l}\text { Enf. } \\
\text { Digestivas }\end{array}$ & $T_{5}$ & \begin{tabular}{l|l}
$\mathrm{I}_{4}$ & $\mathrm{I}_{1}$
\end{tabular} & $\begin{array}{l}\text { I59 años } \\
\text { (DS } 1,97)\end{array}$ & $\left.T_{58,50}\right|_{61}$ & $T_{19,60 \text { años }}$ & T6,33 años \\
\hline$\longdiv { \text { Varios } }$ & $T_{37}$ & \begin{tabular}{l|l} 
& 10
\end{tabular} & $\begin{array}{l}\text { T56,32 años } \\
\text { (DS } 2,31\end{array}$ & \begin{tabular}{l|l} 
I5,46 & 57,84
\end{tabular} & T 18,39 años & T9,56 años \\
\hline
\end{tabular}

Fuente: Servicio de Prevención y Salud Laboral Diputación Provincial de Cádiz 
Las enfermedades que causan mayor porcentaje de incapacidades permanentes en la población estudiada son:

- Enfermedades osteomusculares: 50 (24,15\%)

- Enfermedades cardiovasculares: 49 (23,67\%)

- Enfermedades neoplásicas: 37 (17,87\%)

- Enfermedades psiquiátricas: 19 (9,18\%)

En todos los grupos de enfermedades el porcentaje de expedientes resueltos fue mayor en hombres, a excepción de las enfermedades osteomusculares y las enfermedades psiquiátricas donde prevalece el porcentaje de mujeres.

La distribución de las causas de incapacidad por sexos fue la siguiente:

\begin{tabular}{|ll|}
\hline MUJERES & \\
\hline Enf. Osteomusculares & $34(39,08 \%)$ \\
\hline Enf. Neoplásicas & $17(19,54 \%)$ \\
\hline Enf. Cardiovasculares & $14(16,09 \%)$ \\
\hline Enf, Psiquiátricas & $10(11,49 \%)$ \\
\hline HOMBRES & \\
\hline Enf. Cardiovasculares & $35(28,93 \%)$ \\
\hline Enf. Neoplásicas & $20(16,53 \%)$ \\
\hline Enf. Osteomusculares & $16(13,22 \%)$ \\
\hline Enf. Respiratorias & $10(8,26 \%)$ \\
\hline
\end{tabular}

Existe un claro predominio femenino en las enfermedades osteomusculares como causa de incapacidad permanente, mientras que en el caso de los hombres se observa el predominio en el grupo de enfermedades respiratorias y neoplásicas.

En cuanto a la media de edad, en sentido creciente, la distribución de los grupos de enfermedades causantes de incapacidades permanentes fue la siguiente:

- Enfermedades Psiquiátricas: 51,16 años (DS 4,38).

- Enfermedades Neoplásicas: 53,73 años (DS 3,87).
- Enfermedades Osteomusculares: 54,60 años (DS 3,18).

- Enfermedades Respiratorias: 58,45 años (DS 3,43)

La distribución de la media de edad por sexos fue la siguiente:

\begin{tabular}{|ll|}
\hline MUJERES \\
\hline Enf. Psiquiátricas & 46,80 años \\
\hline Enf. Osteomusculares & 50,37 años \\
\hline Enf. Neoplásicas & 51,18 años \\
\hline Enf. Respiratorias & 56 años \\
\hline HOMBRES & \\
\hline Enf. Neoplásicas & 55,93 años \\
\hline Enf. Osteomusculares & 57,63 años \\
\hline Enf. Cardiovascualres & 58,63 años \\
\hline Enf. Respiratorias & 58,91 años \\
\hline
\end{tabular}

En cuanto a la pérdida de años de vida laboral, la distribución de los grupos de enfermedades fue la siguiente:

- Enfermedades Psiquiátricas: 14,18 años

- Enfermedades Neoplásicas: 11,24 años

- Enfermedades Ostemusculares: 9,44 años

- Enfermedades Respiratorias: 7,98 años

En relación con las enfermedades neoplásicas podemos concluir, según los datos obtenidos en la población estudiada, que nos encontramos con una patología que es la tercera causa de incapacidad permanente, que prácticamente afecta por igual a hombres y a mujeres. Igualmente afecta a la población en plena edad productiva, y después de las enfermedades psiquiátricas son las que mas años de pérdida de vida laboral producen

\section{DISCUSIÓN}

Según los datos mas recientes publicados por la Asociación Española contra el Cáncer (AECC) más del $50 \%$ de las personas diagnosticadas de cáncer, en sus diferentes localizaciones y tipos tumorales, sobreviven a lo cinco años de 1 fecha del diagnóstico. 
El binomio tan extendido cáncer-jubilación se plantea como modificable a tenor de las expectativas de vida y con la calidad de la misma que se puede ofrecer a las personas afectadas.

El reciente estudio titulado "El Cáncer de mama: la enfermedad y sus implicaciones laborales", elaborado por la Federación Española de Cáncer de Mama (FECMA), concluye que en el $40 \%$ de las pacientes encuestadas abandonó su empresa, y que el 35\% tuvo problemas para continuar desempeñando su trabajo. El cambio de puesto de trabajo se dio en un $10 \%$ de los casos.

Las mujeres encuestadas manifestaban que sí por parte de la empresa se hubiesen tenido en cuenta sus características especiales, y se hubiesen propuesto modificaciones en sus condiciones de trabajo (tipo de tareas, horarios flexibles, reducción horaria, etc) posiblemente hubiesen podido continuar desarrollando su actividad laboral.

Las dificultades para continuar trabajando después del diagnóstico de cáncer se dan más en el sector privado frente al sector público. (73 y 53\% respectivamente).

Como no podía ser de otra manera, el tipo de actividad profesional y la "red de apoyo" con la que cuenta la paciente fueron fundamentales para compatibilizar la enfermedad con la actividad laboral.

La localización tumoral influye en la posibilidad de que la persona pueda seguir desarrollando su actividad profesional. Los tumores de piel, mama y aparato genital femenino, glándula tiroides $\mathrm{y}$ aparato urinario son los que con mayor porcentaje compatibilizan la continuación de la capacidad laboral (8).
Las mayores tasas de supervivencia a los cinco años en hombres se consiguen en los tumores de tiroides, testículo y vejiga $(82 \%, 75 \%$ y $75 \%$ respectivamente). En las mujeres a los cinco años la mayor supervivencia se consigue en tumores como el melanoma, de tiroides y de laringe $(90 \%, 86 \%$ y $80 \%$ respectivamente) (9).

En una empresa, hipotética, de alrededor de 1800 trabajadores con una distribución equilibrada por sexos (50\% de cada sexo) se pueden esperar anualmente entre 6 y 7 pacientes a los que el sistema sanitario puede diagnosticar de cáncer (10). Corresponderá a la medicina y a la enfermería del trabajo valorar si las capacidades del trabajador se ven mermadas como para iniciar un expediente de incapacidad permanente. La mayor parte de los pacientes pasan gran parte del tratamiento en su domicilio. Una correcta adaptación del puesto de trabajo, en aquellos casos en que sea técnica y organizativamente posible, y una correcta colaboración por parte de las Unidades de Salud Laboral pueden hacer recuperables para el mercado laboral a una buena parte de esta población trabajadora.

Estudios, no definitivos, concluyen que la permanencia en la actividad laboral, con ciertas limitaciones, de los pacientes diagnosticados de cáncer, aumentan sus expectativas de vida y mejoran su capacidad funcional para actividades de la vida diaria (11).

Es preciso contar con instrumentos que nos permitan medir la calidad de vida de los pacientes-trabajadores con cáncer para poder determinar aquellos a los que se les puede plantear el poder continuar con su actividad laboral una vez diagnosticada y tratada correctamente la enfermedad (12). 


\section{BIBLIOGRAFIA}

1. López Abente G y cols. La situación del cáncer en España. Ministerio de Sanidad y Consumo. Madrid 2003. http://www.cneiscii.es

2. Ferlay J, Bray F, Pisan P, Parkin DM. GLOBOCAN 2002: Cancer Incidente, Mortality and Prevalence Worldwide. IARC CancerBase ${ }^{\circ} 5$, version 2.0 Lyon IARC Press 2004. http://www-dep.iarc.fr

3. INE (Instituto Nacional de Estadística) Movimiento Natural de la Población. Defunciones según causas de muerte. Años correspondientes. http://www.ine.es

4. Hartunian NS, Smart ChN, Thompson MS. The incidence and economic costs of cancer, motor vehicle injuries, coronary heart disease and stroke: a comparative analysis. AM J Public Health 1980; 70: 1249-60.

5. López Bastida J, Serrano Aguilar P, Duque González B. Los coste socioeconómicos d elas enfermedades cardiovasculares y del cáncer en las Islas Canarias en 1998. Gaceta Sanitaria 2003; 17. 3

6. Kiiskinen U, Vartiainen E, Pekurinen M, Puska P. Does prevention of cardiovascular diseases lead to decreased cost of illness? Twenty years of experience from Finland. Prev Med 1997; 26: 220-6.
7. Chan B, Coyte P, Heick C. Economic impact of cardiovascular disease in Canada. Can J Cardiol 1996; 10: 255-9.

8. Alonso Sordo J, Santiago González C, Jiménez del Val D, Palacin Rojo J, Royo Sánchez C, Urbina Torija J, Bárcena Marugán A. GRUPO PREVALCAN de Guadalajara. Situación clínica y funcional de los pacientes con antecedentes de cáncer. SEMERGEN 2004 (30) 11: 542547

9. La situación del cáncer en España. Madrid. Ministerio de Sanidad y Consumo. 2005. http://www.msc.es/

10. Molero García Jm. El cáncer. Actualización en Medicina de Familia. 2006. vol 2(4): 183-184.

11. Feuerstein M, Harrington CB. Recommendations for the US National Occupational Research Agenda: Research on cancer survivorship, musculoskeletal disorders and work disability. Journal of Occupational Rehabilitation. 2006. vol 16 (1): 1-5

12. Agra Varela A, Badía Llach X, Gil Miguel A. Instrumentos para la medición de la calidad de vida en pacientes con cáncer. Medicina Clínica (Barcelona) 1998. 110 (18): 703-708. 